\title{
Intermittent delta waves mimics inferior myocardial infarction in young athlete
}

\author{
Massimo Bolognesi* \\ Centre for Sports Cardiology - Internal General Medicine, Ausl della Romagna - Cesena Italy
}

\begin{abstract}
WPW syndrome is recognized by the existence of delta waves and a short PR interval on the electrocardiogram. Delta waves may appear as a positive or negative deviation on ECG tracing corresponding to the localization of accessory pathway. Wolff-Parkinson-White (WPW) syndrome may exist in healthy athletes. Herewith the author describes a case report of a young athlete who showed an ECG-WPW pattern that mimics an inferior myocardial infarct. This article illustrates this ECG finding and provides helpful clues for arriving at the correct diagnosis.
\end{abstract}

\section{Introduction}

Electrocardiograms (ECGs) are the most powerful tool to detect anomalies in athletes, even if they're considered healthy people [1]. ECGs of patients with Wolff-Parkinson-White (WPW) syndrome may exist in healthy athletes [2]. WPW syndrome is recognized BY the existence of delta waves and a short PR interval on the electrocardiogram. Delta waves may appear as a positive or negative deviation on the ECG corresponding to the localization of accessory pathways. Sometimes the WPW-ECG pattern may simulate other pathologic states such as myocardial infarction, ventricular bigeminy, accelerated idioventricular rhythm, or electrical alternates [3]. Sports pre-participation screening in a young athlete is going to become a challenge for sport cardiology doctors who practice every day with the knowledge of the benefits and harms of their intervention because no one knows if pre-participation screening of athletes is effective in reducing the risk of sudden death [4].

\section{Case report}

A 19-year old male athlete came to our center for sports cardiology to undergo pre-participation screening for volleyball. His family history was unremarkable and physical examination resulted within normal limits. No cardiovascular risk factors were present and he was asymptomatic for chest pain, syncope, and palpitations. Spirometry and urinalysis were normal. He was asymptomatic. Twelve-lead ECG at rest showed a sinus rhythm with frequency variation (respiratory sinus arrhythmia) with a heart rate of $92 \mathrm{bpm}$; PR $118 \mathrm{~ms}$ (short), QRS of $116 \mathrm{~ms}$ (nonspecific enlargement); normal axis and the presence of abnormal Q waves in the inferolateral leads (like pseudo necrosis). Electrocardiographic pattern was suggestive of ventricular pre-excitation due to left posterior-septal pathway, which simulates inferior myocardial features (Figure 1). An exercise OF stress testing was normal with stable pre-excitation ECG pattern. Subsequently, a Trans-Thoracic Echocardiography showed normal morphological and functional heart findings. Consultation with EP Cardiology confirmed the suspicion that this ECG represents a Pseudo-Infarction Pattern in a patient with WPW. Electrophysiological study was not performed in this asymptomatic athlete.

\section{Discussion}

The ECGs of WPW syndrome may mimic myocardial infarct patterns. If the delta wave is oriented superiorly, it may register as an abnormal $\mathrm{Q}$ wave in the inferior leads, and the tracing can be mistaken for an inferior myocardial infarct. Literature reported that among 44 patients with WPW syndrome referred to their institution, $31(70 \%)$ had negative delta waves in one or more leads simulating infarct pattern [5]. In the setting of sports pre-participation screening there are many questions to ask: a) How should a sports physician interpret this tracing? b) What clinical entities should be considered in the differential diagnosis? c) How might one proceed in that evaluation? The evidence of an abnormal 12-lead ECG for this young adult should raise several considerations (i.e., previously undetected congenital heart disease; some type of cardiomyopathy; a prior episode of myocarditis that spontaneously resolved; etc.). Anyhow, an echocardiogram was obtained, looking for underlying structural heart disease. To make this case more difficult, everyone must know that the appearance of WPW on the ECG may be varied and subtle. In fact, the inferior pseudo-infarction pattern seen here is due to negative delta waves in leads II and III. Therefore, delta waves are otherwise not readily recognizable on this tracing. In this case, the ECG is markedly abnormal for a 19-year old man. The long lead II rhythm strip at the bottom shows aphasic arrhythmia, with upright $P$ waves with a fixed PR interval preceding each QRS complex. This is most likely marked sinus arrhythmia, but the rhythm is benign in a 19-year old man. It is the remainder of the ECG that is most exciting. All intervals (PR, QRS, and QT) appear to be normal. The mean THE QRS axis is indeterminate (predominantly negative in both leads I and aVF). What

Correspondence to: Massimo Bolognesi, Centre for Sports Cardiology - Internal General Medicine, Ausl della Romagna, Via Ungaretti 494 47521, Cesena- Italy, Tel: +390547645074; E-mail: massbolo1@tin.it

Key words: delta wave, WPW syndrome, electrocardiogram, athletes, myocardial infarction

Received: October 27, 2016; Accepted: November 23, 2016; Published: November 28, 2016 


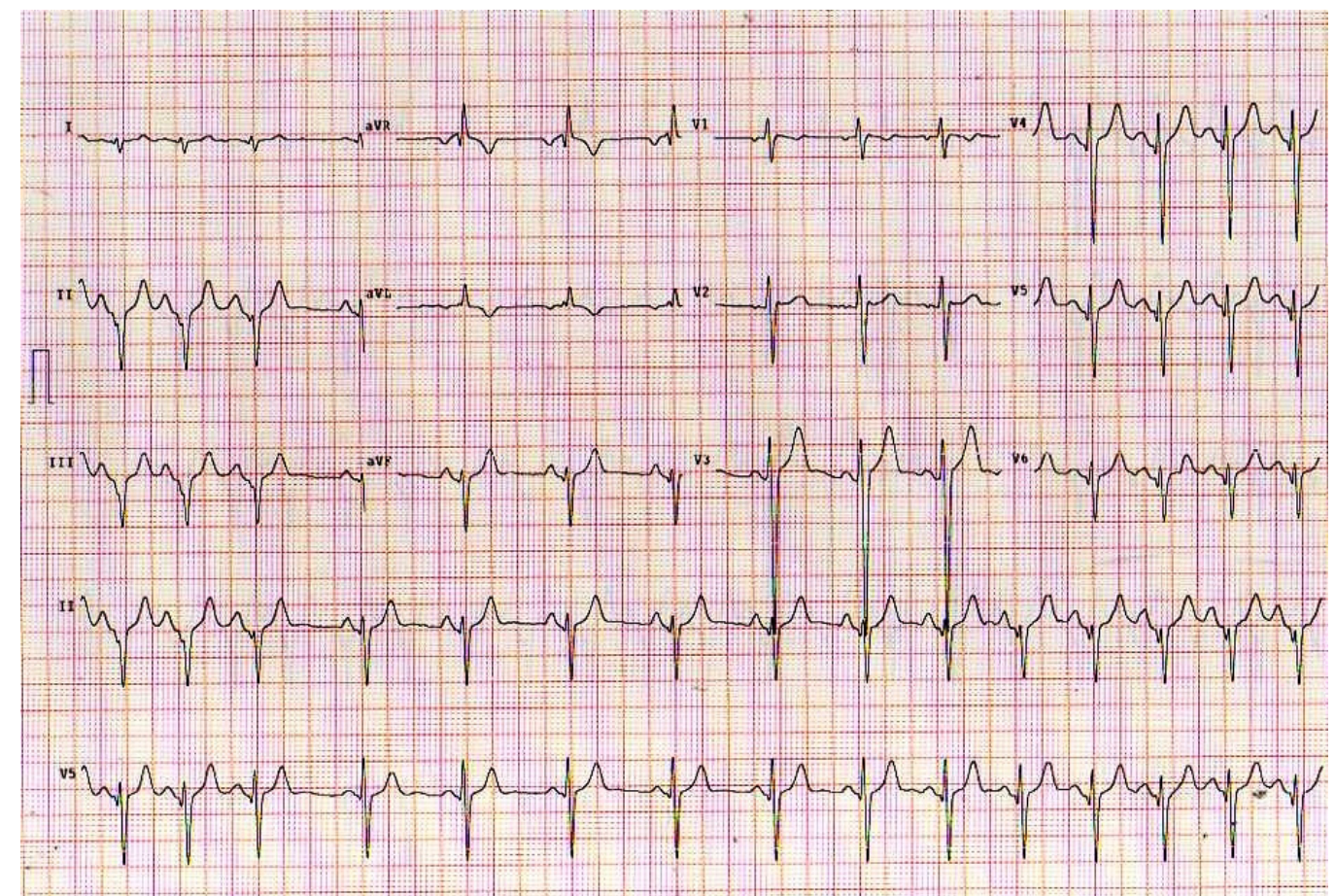

Figure 1. 12-lead ECG and long lead II - V5 rhythm strip obtained from an asymptomatic, young adult man as part of a sports pre-participation screening evaluation.

is very interesting and strange - is inferior negativity of the QRS and an almost reverse $\mathrm{R}$ wave progression in the chest leads with small $\mathrm{q}$ waves in leads V4, V5, V6. There is a T-wave inversion in lead aVL, with T-waves otherwise upright. An untrained eye might argue that in this ECG does not present a clear delta wave and that the long strip may show a striking appearance for a wandering pacemaker. In fact, it is not, because a closer reading shows an intermittent delta wave on the long strip in D2. Indeed, in Figure 1 we can see subtle-but-real down-sloping of the negative delta wave that is intermittently present to varying degrees in lead II! Over the course of the next several beats, this q wave deepens and widens - eventually developing subtle down-sloping toward the end of the $\mathrm{P}$ wave for the last few beats in the tracing. They are unmistakable in this 19-year old diagnosed by EP to have WPW when you compare normal conduction with P-QRS appearance at the beginning and at the end of this tracing. Thus, after the first 3 beats, that are conducted over the AP (Accessory Pathway) - we are seeing several normally conducted beats (beats 4,5,6) - and then progressive shortening of the PR interval with the corresponding widening of the QRS complex as a delta wave again develops after the middle of this long lead II rhythm strip. This cyclic pattern, with a gradual change in the relative amount of pre-excitation that we see here in this patient with newly diagnosed WPW, is known as the Concertina Effect. The interesting news is that when we see a Concertina Effect (an unusual phenomenon in patients with WPW) it is a good indicator of a relatively long AP refractory period since a longer refractory period limits the number of impulses that can be transmitted by the AP. This portends a relatively lower risk of sudden death during a WPW-related tachyarrhythmia. Thus, recognition of the Concertina Effect in this 19-year old with newly diagnosed WPW at least provides positive prognostic information. In conclusion, besides that this is not a "normal" ECG for an otherwise healthy 19-year old, he was considered suitable for sports participation for the absence of arrhythmias risk!

\section{Conclusion}

There are pros and cons to pre-participation ECG evaluation of otherwise healthy young adult athletes. The obvious goal of such evaluation is to detect occult cardiac conditions that might prove hazardous if unrestricted physical exertion is allowed. Careful investigation of surface ECG is necessary for sports physicians to avoid incorrect diagnosis.

\section{Conflict of interest}

None

\section{References}

1. Lawless CE, Best TM (2008) Electrocardiograms in athletes: interpretation and diagnostic accuracy. Med Sci Sports Exerc 40: 787-798. [Crossref]

2. Rao AL, Salerno JC, Asif IM, Drezner JA (2014) Evaluation and management of wolffParkinson-white in athletes. Sports Health 6: 326-332. [Crossref]

3. Wang K, Asinger R, Hodges M. Electrocardiograms of Wolff-Parkinson-White syndrome simulating other conditions American Heart Journal Volume 132, Issue 1, Part 1, July 1996

4. Semsarian C, Ingles J (2016) Preventing sudden cardiac death in athletes. BMJ 353 i1270. [Crossref]

5. Ruskin JN, Akhtar M, Damato AN, Ticzon AR, Lau SH, et al. (1976) Abnormal Q waves in Wolff-Parkinson-White syndrome. Incidence and clinical significance. JAMA 235: 2727-2730. [Crossref]

Copyright: (C)2016 Bolognesi M. This is an open-access article distributed under the terms of the Creative Commons Attribution License, which permits unrestricted use, distribution, and reproduction in any medium, provided the original author and source are credited. 